\title{
Integración de herramientas para el control de gestión. Análisis de un caso de estudio
}

\section{(Integration of management control tools. Analysis of a case study)}

\author{
Raúl Comas Rodríguez ${ }^{1}$, Dianelys Nogueira Rivera², Félix Romero Bartutis ${ }^{3}$, \\ Marisdany Lumpuy Rodríguez ${ }^{3}$
}

\begin{abstract}
Resumen:
El objetivo del artículo es diseñar e implementar un procedimiento que integre herramientas de control de gestión con enfoque de procesos, que contribuya a mejorar la eficiencia y la eficacia de la gestión empresarial. Se lleva a cabo un estudio experimental donde se define un procedimiento, basado en un cuadro de mando integral, que integra la gestión por procesos desde la planeación estratégica y su evaluación. Como resultados del trabajo se definen los factores clave de éxito de la empresa asociados a las cuatro perspectivas del cuadro de mando integral las cuales se enlazan a través de relaciones causa-efecto y se obtiene el mapa estratégico que permite visualizar y comunicar la estrategia de la empresa. Los indicadores para medir la actuación de los factores claves de éxito se integran con los procesos y se evalúan con la asistencia de un software. Con la implementación del procedimiento en una empresa comercializadora, se integró la definición de los procesos desde la propia planificación estratégica, se evaluó su alineamiento, y los indicadores de eficiencia y eficacia evaluados mejoraron su desempeño.
\end{abstract}

Palabras clave: estrategia, control de gestión, cuadro de mando integral, procesos, alineamiento estratégico

\begin{abstract}
:
The objective of this article is to design and to implement a procedure that integrates management control tools focusing on process, to improve the efficiency and the efficacy. It was carried out an experimental study where is defined a procedure, based in the Balanced Scorecard, which integrates the process management into the strategic planning and their evaluation. As results of this work, we define the key factors of success associated with the four perspectives of the Balanced Scorecard that are linked through the cause-effect relations obtaining the strategic map that allows visualizing and communicating the enterprise strategy. The indicators evaluate the key factor of success, integrating the process with the assistance of a software. The implementation of the procedure in a commercialization enterprise contributed to integrate the process definition into the strategic planning. The alignment was evaluated and the efficiency and efficacy indicators improved the company's performance.
\end{abstract}

Keywords: strategy, management control, balanced scorecard, process, strategic alignment

\footnotetext{
${ }^{1}$ Universidad Técnica de Ambato, Ambato - Ecuador ( raul.comas@uta.edu.ec )

2 Universidad de Matanzas, Matanzas - Cuba ( dianelys.nogueira@umcc.cu )

${ }^{3}$ Universidad de Sancti Spíritus, Sancti Spíritus - Cuba ( \{felixr, mlumpuy\} @uniss.edu.cu )
} 


\section{Introducción}

El sistema económico, político y social del mundo es complejo, y para las organizaciones es un reto dar respuesta a sus necesidades en tiempos turbulentos (Boada Grau \& Gil Ripoll, 2009; Del Canto, 2011). En la actualidad, las organizaciones, motivadas por los cambios socioeconómicos, reclaman la incorporación de nuevos métodos que les permitan gestionar esfuerzos, recursos, capacidades y adecuarse a las exigencias del entorno para ganar eficiencia y eficacia en sus procesos (Paladino \& Williams, 2008; Shpilberg, Berez, Puryear, \& Shah, 2007).

La dirección estratégica es la forma más aplicada de gestionar las organizaciones, por su carácter abarcador, que integra en un solo pensamiento estratégico todos los sistemas. En el momento de desplegar la estrategia diseñada o gestionar sus procesos, las organizaciones suelen fracasar porque carecen de un sistema de gestión capaz de integrar y alinear ambos elementos. (Agudelo Tobón \& Escobar Bolívar, 2010; Amo Baraybar, 2010; Borchardt, Alfonso Sellito, \& Medeiros Pereira, 2007; Kaplan \& Norton, 2008)

Por tanto, a fin de contribuir en las investigaciones sobre el tema, el propósito de este trabajo es desarrollar un procedimiento que integre herramientas del control de gestión con enfoque de procesos, apoyado en un sistema de información que contribuya a evaluar la eficiencia y la eficacia en el sistema empresarial.

Se plantea como hipótesis de la investigación: el desarrollo de un procedimiento que permita integrar herramientas de control de gestión con enfoque de procesos contribuye a evaluar la eficiencia y eficacia en el sistema empresarial.

Para la aplicación del procedimiento se toma como caso de estudio principal una empresa comercializadora donde la aplicación del procedimiento propuesto demuestra la factibilidad de su aplicación y sus resultados contribuyen a la mejora de la misma.

\section{Metodología}

Para la aplicación del procedimiento que integra herramientas de control de gestión con un enfoque de procesos se deben cumplir previamente con los siguientes requisitos:

1. Apreciar en la organización la necesidad del cambio y el compromiso con la aplicación de los resultados.

2. Que exista la formación del personal implicado en las transformaciones culturales a desarrollar.

3. La dirección estratégica como filosofía asumida por la alta dirección.

4. Un clima organizacional que contribuya al trabajo en equipo, un ambiente de aprendizaje permanente y acceso a la información. 


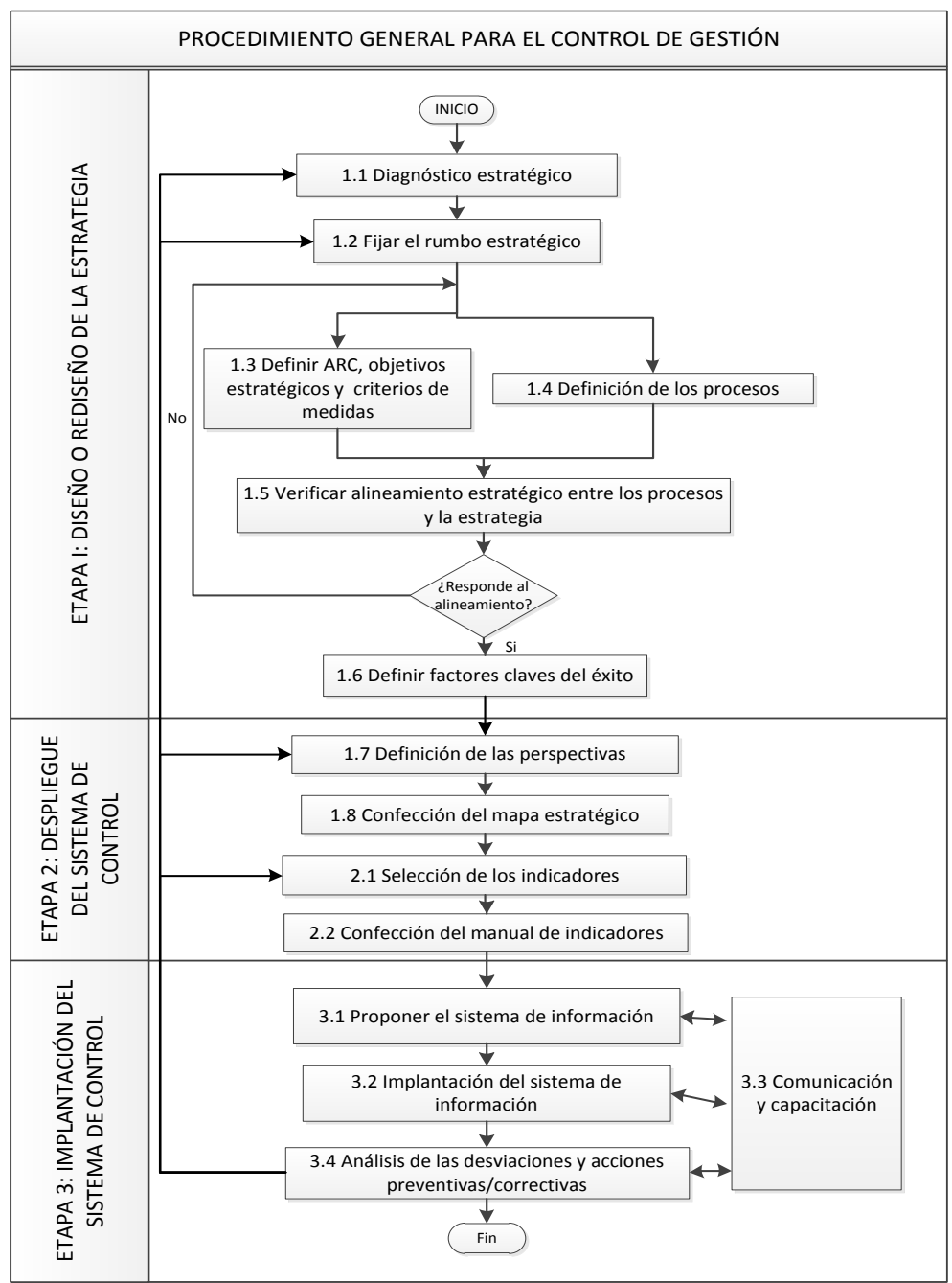

Figura 1. Procedimiento para el control de gestión.

En la Figura 1 se muestra el procedimiento propuesto que consta de tres etapas que incluyen: el diagnóstico organizacional, la definición o redefinición de la estrategia empresarial y la definición de los procesos, el despliegue del cuadro de mando integral (CMI), y su implementación y control.

\section{Etapa I. Diseño o rediseño de la estrategia}

Como primer paso para la definición de cualquier estrategia de negocio es importante y necesario un diagnóstico estratégico de la organización. Para la realización del diagnóstico se obtiene el compromiso real de la dirección porque todo proyecto de esta envergadura, sin un verdadero liderazgo de los directivos y su apoyo, resulta infructuoso. Se conforma un equipo de trabajo para ampliar la base de deliberaciones y consenso, compuesto entre seis y doce personas, encargados de la definición del diagnóstico. Para obtener los resultados del diagnóstico estratégico se propone un análisis DAFO.

A partir de los resultados de este diagnóstico, se fija el rumbo estratégico de la organización como punto de partida de la definición de la estrategia. Es necesario fijar el rumbo estratégico de la organización, con todas sus variables de salida: misión, visión, valores, estrategias, objetivos y 


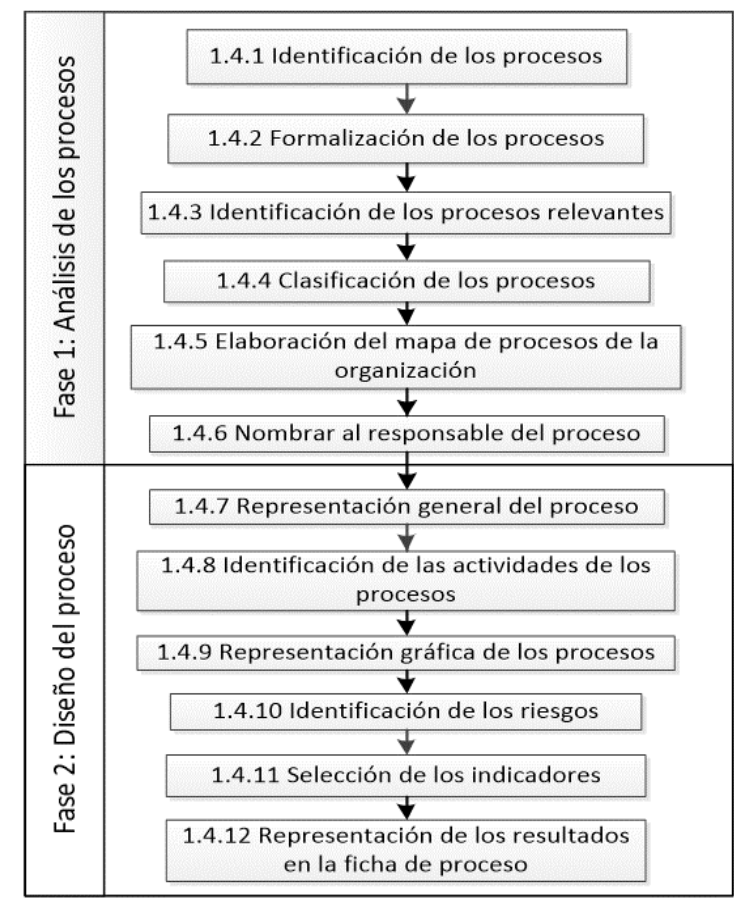

Figura 2. Procedimiento para la definición de los procesos. empresariales.

políticas. Las áreas de resultados claves (ARC) son simplemente áreas o categorías esenciales para el rendimiento efectivo en la institución o empresa. Para cada ARC se deben definir los objetivos estratégicos que no son más que las declaraciones de las aspiraciones a alcanzar en el período determinado. Cada objetivo estratégico debe contar con sus criterios de medidas para evaluar su cumplimiento.

Para determinar los procesos se implementa el procedimiento que se muestra en la Figura 2. Al aplicar el procedimiento se obtiene:

- El mapa que integra los procesos de la empresa, clasificados en: estratégicos, operativos y de apoyo y su interrelación.

- Un diagrama de cada proceso que muestra una explicación detallada de cada una de las actividades del proceso y quién lo hace.

- Una ficha para cada proceso, que representa un soporte informativo de las características relevantes para el control de todas las actividades: responsable, objetivos, descripción, recursos necesarios, documentación, procesos con los cuales se relaciona, principales riesgos e indicadores para evaluar su eficacia.

Después de definidas las categorías básicas de la estrategia e identificados los procesos de la organización es necesario contrastarlas en busca de evaluar el alineamiento estratégico entre los procesos y los objetivos estratégicos. Una de las mayores debilidades en las estrategias actualmente es la falta de alineamiento entre las propuestas definidas a largo plazo (establecidas a través de los objetivos estratégicos) y su accionar, en el corto plazo, en los procesos que se ejecutan en la organización. Para evaluar la relación se realizan los siguientes pasos: 
1. Crear matriz de impacto entre los procesos y los objetivos estratégicos. Se evalúa el impacto de los procesos en los objetivos estratégicos con la evaluación de 1 (bajo) a 10 (alto) puntos.

2. Calcular el peso relativo de los objetivos estratégicos y los procesos. Para el cálculo de los pesos relativos se puede utilizar el método de las jerarquías analíticas (AHP) ${ }^{4}$.

3. Calcular el índice de alineamiento de los objetivos estratégicos a través de la Ecuación 1.

$$
I a o=\sum_{k=1}^{n}\left(O E_{k} * \omega O_{k}\right)
$$

Dónde:

lao: índice de alineamiento de los objetivos estratégicos

$O E_{k}$ : media de los impactos de los procesos en el objetivo estratégico k.

$\omega o_{k}$ : peso relativo del objetivo estratégico $\mathrm{k}$.

$\mathrm{n}$ : cantidad de objetivos estratégicos.

4. Calcular el índice de alineamiento de los procesos a través de la Ecuación 2.

$$
\operatorname{Ipr}=\sum_{k=1}^{m}\left(P R_{k} * \omega p_{k}\right)
$$

Dónde:

Ipr: Índice de alineamiento de los procesos.

$P R_{k}:$ media de los impactos del proceso k en los objetivos estratégicos.

$\omega p_{k}$ :peso relativo del proceso $\mathrm{k}$

m: cantidad de procesos

5. Evaluar la posición de los objetivos estratégicos y los procesos en la matriz de impacto procesos/objetivos estratégicos. (Figura 3). En caso de ubicarse en el cuadrante superior derecho existe alineamiento entre los objetivos estratégicos y los procesos. En caso de ubicarse en el cuadrante superior izquierdo los objetivos estratégicos de mayor peso no son impactado por los procesos. En caso del cuadrante inferior izquierdo indica que los procesos de mayor peso no impactan en los objetivos estratégicos. En el cuadrante inferior izquierdo es necesario el rediseño de la estrategia y los procesos porque no hay alineamiento.

\footnotetext{
${ }^{4}$ El método de las jerarquías analíticas, propuesto por el matemático Thomas L. Saaty en 1980, es un método para abordar problemas que tienen un número finito de soluciones; formula el problema de decisión permitiendo organizar la información respecto al problema de decisión, y que dicha información sea descompuesta y analizada en su diferentes partes, para elegir la mejor alternativa o solución.
} 


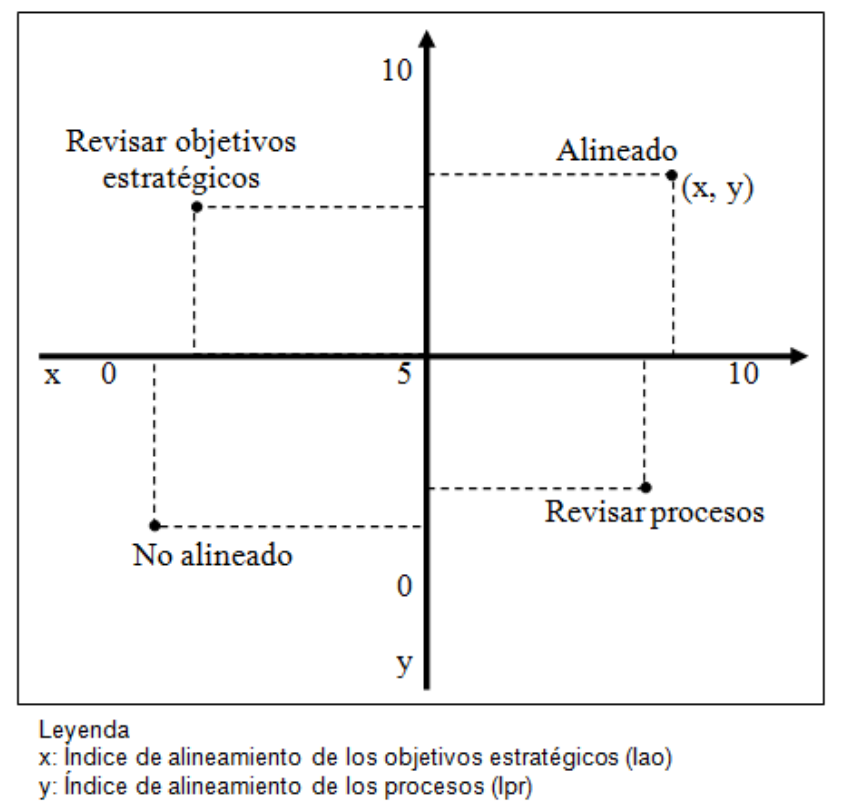

Figura 3: Matriz de impacto procesos/objetivos estratégicos.

Para finalizar la fase se definen los factores claves del éxito (FCE) que se identifican para concentrar los esfuerzos y recursos de la organización en ellos. Los FCE deben tener una correspondencia directa con los objetivos estratégicos definidos (Comas Rodríguez, 2013).

\section{Etapa II. Despliegue del sistema de control}

Definido el rumbo estratégico, se despliega el CMI como herramienta de control de gestión, mediante la cual se puede: medir y evaluar la gestión empresarial; tener una visión global de la organización, y apoyar el proceso de toma de decisiones, se conjugan los indicadores financieros y no financieros en diferentes perspectivas a través de las cuales es posible analizar la empresa en su conjunto.

Kaplan y Norton propusieron en la confección del CMI la utilización de cuatro perspectivas: financiera, clientes, procesos internos, y aprendizaje y crecimiento. Se puede comenzar a trabajar en el montaje de las perspectivas desde estas cuatro propuestas pero, a partir de las interioridades de cada empresa, se puede definir nuevas acordes a sus características.

Un mapa estratégico es una descripción integrada y lógica de la forma en que se llevará a cabo una estrategia, indica las relaciones causa-efecto relativa a los recursos y capacidades de la empresa que deben llevar a los resultados estratégicos deseados. 
Definir el mapa estratégico de una organización es una actividad compleja. El trabajo de establecer las relaciones causa efecto lleva tiempo y dedicación del equipo de trabajo seleccionado. En la literatura consultada (más de 700 artículos descargados de Internet, y otras referencias) no se dispone de un procedimiento o metodología que guíe como realizarlo. Se propone el procedimiento específico que se muestra en la Figura 4 para la elaboración de un mapa estratégico.

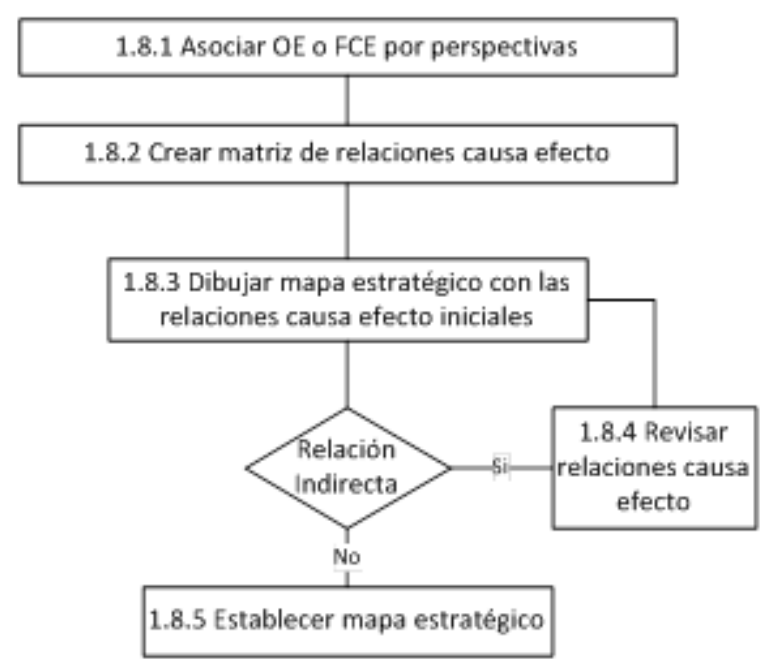

Figura 4. Procedimiento específico para confeccionar el mapa estratégico.

Para comenzar a confeccionar el mapa estratégico se asocian los objetivos estratégicos (OE) o factores claves de éxito a las perspectivas seleccionadas. Se crea una matriz donde por las filas y las columnas se insertan los OE o FCE ordenados por las cuatro perspectivas y se impacta cada uno con el resto en la búsqueda de las relaciones causales que existen entre ellos.

En un lienzo se dibujan los OE o FCE y se relacionan a partir de las relaciones prestablecidas en la matriz. Al dibujarse la primera variante del mapa estratégico es posible visualizar aquellos nexos que resultan secundarios y se detectan los posibles errores existentes en la operacionalización de la estrategia definida. Después de depurar el mapa inicial se mejora la configuración del gráfico propuesto y queda establecido el mapa estratégico final de la organización.

El CMI se fundamenta en la filosofía “...si no puedes medirlo, no puedes gestionarlo". Claramente, algunas operaciones (beneficios, ventas, etcétera) son más fáciles de medir que otras (habilidades de los trabajadores, imagen, etcétera); sin embargo, no por ello debe ser excluidas del CMI, en todo caso puede quedar como "pendiente a desarrollar", pues casi siempre existe un indicador, aunque no sea perfecto. En la presente investigación se propone la evaluación de los indicadores en el CMI en cuatro valores excelente (azul en el CMI), bien (verde), regular (amarillo) y mal (rojo)

Un excelente CMI deberá poseer una variación adecuada de resultados (indicadores efectos) y de inductores de la actuación (indicadores causa) que se hayan adaptado a indicadores de la 
estrategia de la unidad de negocio. Los indicadores del resultado sin los inductores de la actuación no comunican la forma en que conseguirán los resultados, ni proporcionan una indicación temprana de sí la estrategia se propone en práctica con éxito.

Como resultado de esta etapa se obtienen los indicadores necesarios para medir la actuación de los factores clave en cada una de las cuatro perspectivas, con el fin de determinar el grado de consecución de los objetivos estratégicos.

Por último, se confecciona un manual que sirva de consulta y herramienta de trabajo para los directivos y especialistas, el cual debe incluir la información de los indicadores a tener en cuenta: perspectiva, FCE, vínculo con procesos, vínculo con objetivos estratégicos, código del indicador, objetivo del indicador, forma de cálculo, unidad de medida, periodicidad, niveles de referencia, metas concebidas, mejoramiento histórico del indicador (para establecer tendencias), mejor valor logrado para dicho indicador, fecha de entrada al CMI, fecha de salida del CMI y responsable

\section{Etapa III. Implementación y control}

Después de definir el CMI, se trabaja en su implementación, la cual parte de la confección del sistema de información que sirva a la organización para el cumplimiento de los objetivos propuestos y el análisis de las desviaciones y acciones correctivas necesarias en su desempeño.

Un sistema de información para ejecutivos ${ }^{5}$ permite presentar la información en forma rápida y sencilla y, usualmente, posee las características siguientes:

- Tienen los indicadores relevantes.

- Admiten la condensación de la información y su investigación.

- Hay señales de alarma (semáforos) que señalan los desvíos importantes y permiten el control por excepción.

- Posibilitan la visualización gráfica.

- Presentan información interna y externa.

La conformación de los cuadros de mando de los directivos se debe conformar en cascada. Cada responsable de un indicador actualiza sus datos y con ellos se confecciona el cuadro de mando de cada unidad estratégica de negocios y cada gerencia funcional. De todos los indicadores seleccionados en la empresa, se escogen aquellos que son de interés de la alta dirección y con ellos se conforma su cuadro de mando.

El CMI, aunque, normalmente, va dirigido a la dirección de la unidad de negocios donde se implemente, debe ser compartido con todos los trabajadores de la organización. En consecuencia,

5 Denominado en la literatura de habla inglesa Executive Information Systems (EIS). 
su elaboración implica un esfuerzo de comunicación explícita e implícita adicional al normal, pues todas las etapas representan un proceso educativo muy valioso para todos los niveles.

La función de control consiste en la medición del progreso y su comparación con el resultado esperado para que, en caso de que difieran, se tomen las acciones necesarias. Si existieran desviaciones con respecto a las metas establecidas para algún indicador, la dirección debe reunirse con los responsables de los indicadores y tomar las acciones pertinentes para el cumplimiento de los valores fijados.

\section{Resultados}

La implementación del procedimiento se realizó en una organización comercializadora donde se aprovechó el ejercicio de planeación de la empresa para la actualización de la estrategia.

Para el diagnóstico situacional de la organización se creó un equipo de trabajo, encabezado por los directivos de la empresa y en el cual participaron además funcionarios y trabajadores que representan las diferentes áreas de la organización.El objeto social de la organización es amplio y abarca diversas actividades del comercio y los servicios:

- Venta mayorista y minorista de mercancías y combustible

- Venta minorista de servicios gastronómicos

- Prestación de servicios de fotografía y recreación

- Prestación de servicios técnicos en actividades de tecnología, computación y clima.

- Prestación de servicios de instalación y mantenimiento a grupos electrógenos.

- Prestación de servicios de intermediación financiera.

La empresa cuenta con nueve Unidades Estratégicas de Negocio y con ochenta y tres puntos venta con representación en todos los municipios de la provincia, y laboran 719 trabajadores. Para el análisis del entorno y la organización se aplicó una encuesta a una muestra aleatoria del personal para la selección de las oportunidades, amenazas, fortalezas y debilidades; mediante trabajo en equipo se reduce el listado y se obtuvieron los resultados siguientes:

\section{Oportunidades:}

1. Incremento de la demanda por la diversificación de formas de gestión de la propiedad.

2. Incremento de las remesas familiares desde el exterior.

3. Ventas en la red minorista a través de tarjetas de créditos con descuento a colaboradores.

4. Cambios en la política bancaria dirigidos al otorgamiento de créditos a la población.

5. Incremento del marco importador que permite diversificar la oferta

\section{Amenazas:}

1. La crisis económica mundial. 
2. Incremento de la competencia del mercado.

3. Inestabilidad en los suministros de los proveedores nacionales.

4. Carencia de fuerza constructora en la provincia.

5. Regulaciones que limitan la autonomía de la empresa.

\section{Fortalezas:}

1. Capacidad financiera y solvencia económica

2. Desarrollo tecnológico y de infraestructura.

3. Empresa que de forma continua mejora sus procesos y sistemas de gestión.

4. Trabajadores competentes y con experiencia en el trabajo.

5. Credibilidad e imagen.

\section{Debilidades:}

1. Insuficiente uso de los sistemas de estimulación que incide en la baja motivación

2. Incumplimiento con los planes de mantenimiento programados.

3. Insuficiencias en el uso de los sistemas de control y exigencia en las unidades.

4. Limitación en el desempeño de las funciones de los administradores.

5. Sistema de gestión ineficaz para asegurar el reaprovisionamiento oportuno de las mercancías en la red comercial.

Con los datos anteriores se confeccionó la matriz DAFO de la Tabla 1.

Tabla 1. Matriz DAFO.

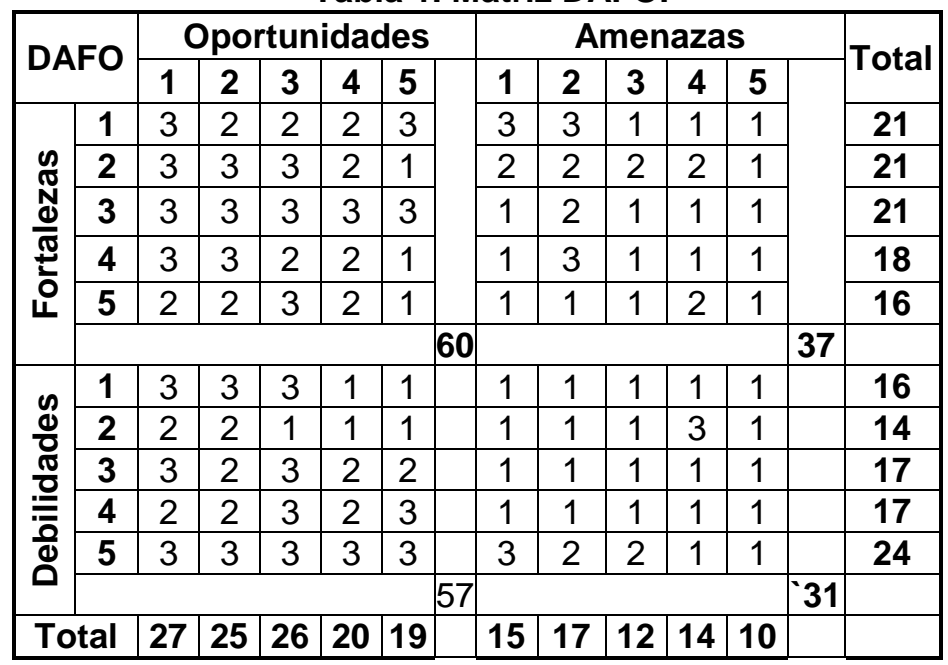

La organización se ubicó en el primer cuadrante, que es característico de las que asumen estrategias ofensivas con énfasis en la eficacia. El análisis DAFO mostró un mayor porcentaje en el primer cuadrante (80\%) por lo que es posible aprovechar los cambios que se avecinan con la actualización del modelo económico, con el apoyo de la capacidad financiera y solvencia económica, el desarrollo tecnológico y de infraestructura, y la mejora continua de los procesos y el sistema de gestión. No obstante al análisis anterior se pudo evidenciar la necesidad de trabajar la 
estrategia en función de atenuar las debilidades presentes que afectan el desempeño de la organización.

Con los elementos declarados en la misión y la visión, y las prioridades en el momento actual se identificaron tres áreas de resultado clave: Gestión económica, Mejora continua y Cumplimiento de la responsabilidad social. Para cada una de las ARC se definieron los objetivos estratégicos con sus criterios de medidas los cuales establecen el vínculo de la misión definida con la visión a alcanzar.

Definida la estrategia se aplicó el procedimiento para la definición de los procesos. En la Figura 5 se muestra el mapa de procesos desplegado. También se definieron los procesos a través de un diagrama de flujo y sus respectivas fichas.

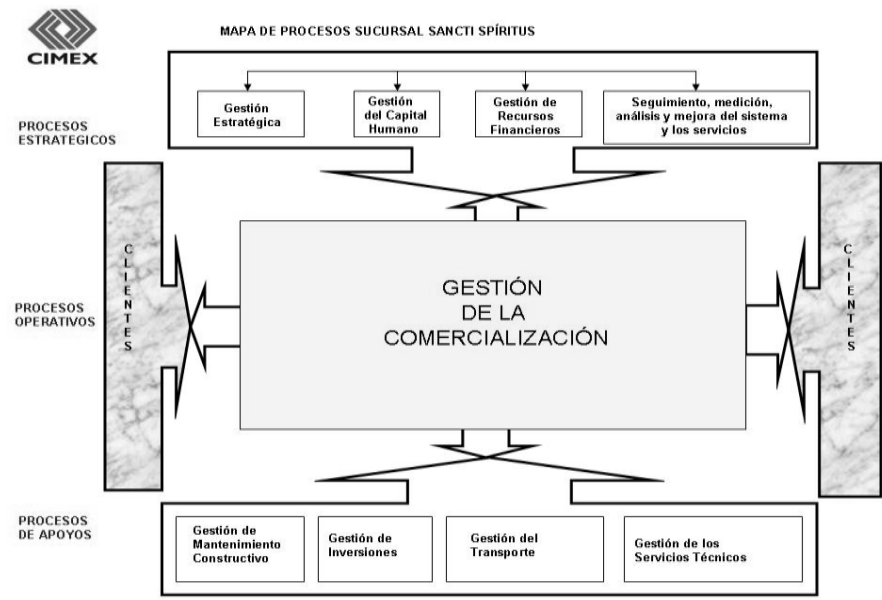

Figura 5. Mapa de procesos

Para verificar el alineamiento estratégico entre los procesos y los objetivos estratégicos se creó la matriz de impacto entre ambos elementos; se calcularon: los pesos relativos a través del método AHP; el índice de alineamiento de los objetivos estratégicos (Tabla 2) y el índice de alineamiento de los procesos con un valor de 7.7631 .

Tabla 2. Cálculo del indicador de alineamiento de los objetivos estratégicos

\begin{tabular}{|c|c|c|c|c|}
\hline & Objetivos estratégicos & Media & $\begin{array}{c}\text { Peso } \\
\text { Relativo }\end{array}$ & \\
\hline 1 & Aumentar de manera sostenida las utilidades & 7.5556 & 0.20911 & 1.5799 \\
\hline 2 & Avanzar en el perfeccionamiento de la gestión & 7.4444 & 0.25083 & 1.8673 \\
\hline 3 & Mantener el AVAL del sistema de gestión de la calidad & 7.5556 & 0.16675 & 1.2598 \\
\hline 4 & Alcanzar un uso más eficiente del Capital Humano & 6.6667 & 0.10729 & 0.7152 \\
\hline 5 & Elevar los resultados en la comercialización & 8.0000 & 0.08769 & 0.7014 \\
\hline 6 & $\begin{array}{l}\text { Mejora de la infraestructura, el mantenimiento } \\
\text { constructivo y tecnológico }\end{array}$ & 8.3333 & 0.05139 & 0.4282 \\
\hline 7 & Consolidar el sistema de Control Interno & 6.8889 & 0.07129 & 0.4911 \\
\hline 8 & Elevar la disciplina financiera & 5.6667 & 0.05564 & 0.3153 \\
\hline \multicolumn{4}{|c|}{ Índice de alineamiento de los objetivos estratégicos } & 7.3586 \\
\hline
\end{tabular}


Para evaluar la posición se creó a matriz de impacto procesos/objetivos estratégicos de la Figura 6. Como resultado de la evaluación se pudo confirmar la existencia de alineamiento entre los objetivos y los procesos por encontrarse el punto de evaluación en el cuadrante superior derecho.

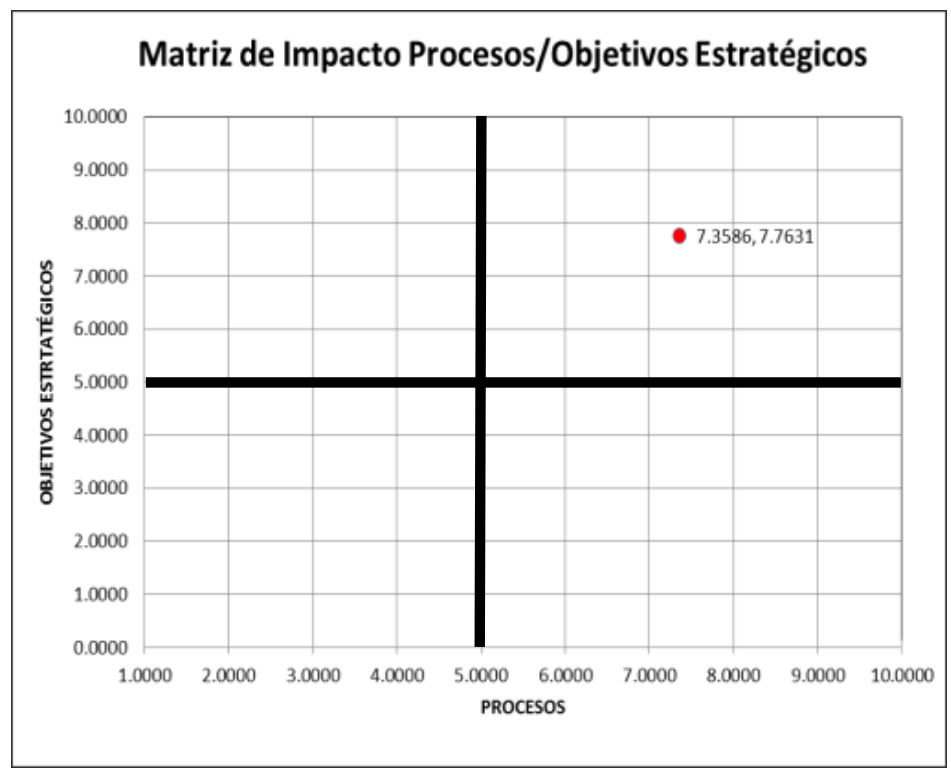

Figura 6. Matriz de impacto procesos/objetivos estratégicos.

A partir del criterio expuesto por los especialistas del equipo de trabajo se identificaron doce factores claves del éxito.

El despliegue del sistema de control comenzó con el montaje de las perspectivas para el CMI. Para la implementación del CMI se trabajó con las cuatro perspectivas definidas por Kaplan y Norton: Financiera, Clientes, Procesos Internos, y Aprendizaje y Crecimiento.

Para definir el mapa estratégico los FCE se reagruparon por perspectivas (Tabla 3) y se creó la matriz de relaciones causa-efecto de los posibles enlaces entre los FCE.

Con los resultados de la matriz de impacto de las relaciones causa efecto se obtuvieron 29 posibles impactos entre los FCE. Se realizó el dibujo inicial de la propuesta del mapa estratégico y una revisión para eliminar los nexos secundarios de los impactos definidos desde su visualización. Como resultado final se obtuvo el mapa estratégico de la organización objeto de estudio (Figura 7).

Para el despliegue del CMI se definieron los indicadores estratégicos que evaluan los FCE y que también tienen su impacto en los objetivos estratégicos y en los procesos. 
Tabla 3. FCE asociados a las perspectivas del CMI.

\begin{tabular}{|c|l|}
\hline Perspectivas & \multicolumn{1}{|c|}{ Factores claves de éxito } \\
\hline \multirow{4}{*}{ Financiera } & Incrementar la rentabilidad \\
\cline { 2 - 2 } & Incrementar ingresos \\
\cline { 2 - 2 } & Reducir costos y gastos \\
\hline \multirow{2}{*}{ Clientes } & Elevar la satisfacción de los clientes \\
\cline { 2 - 2 } Procesos & Mejorar la calidad del servicio \\
\cline { 2 - 2 } Internos & Incrementar la gestión de ventas \\
\cline { 2 - 2 } & Minimizar riesgos \\
\hline \multirow{3}{*}{$\begin{array}{c}\text { Aprendizaje y } \\
\text { Crecimiento }\end{array}$} & Elevar la productividad de los trabajadores \\
\cline { 2 - 2 } & Aplicar sistemas de pago por resultados \\
\cline { 2 - 2 } & Mejorar las competencias laborales \\
\cline { 2 - 2 } & Mantener y renovar la infraestructura constructiva y tecnológica \\
& \\
\hline
\end{tabular}

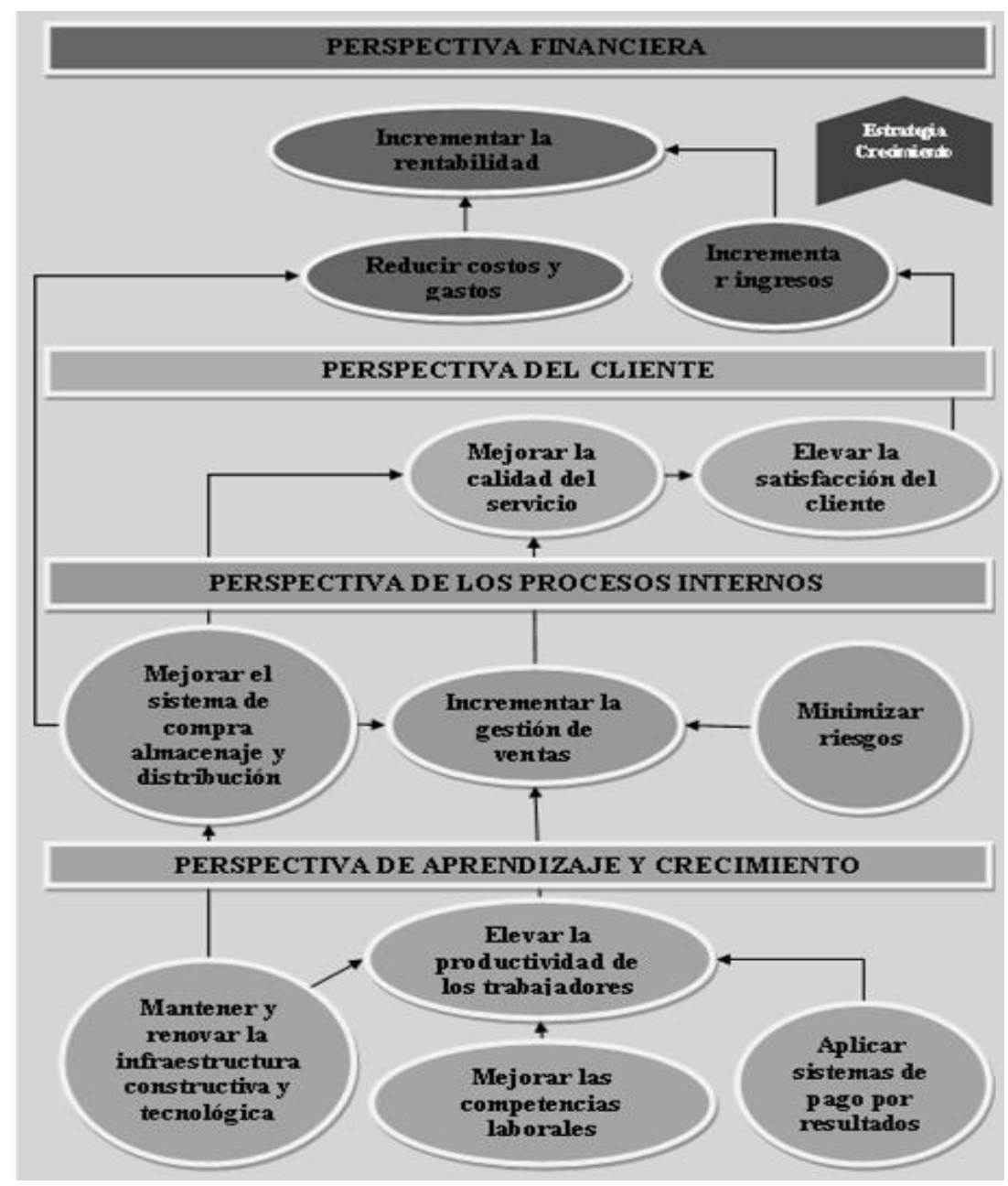

Figura 7. Mapa estratégico 
La Tabla 4 muestra el cuadro de mando de la Dirección General al cierre del primer trimestre, en el momento se reportaron deficiencias en la evaluación de cuatro indicadores: el nivel de satisfacción de los pedidos, el índice de ventas de mercancías por metros cuadrados, la correlación salario medio productividad y el cumplimiento del plan de mantenimientos a equipos tecnológicos. Otros seis indicadores se encuentran evaluados de regular.

Tabla 4. Cuadro de mando de la Dirección General.

\begin{tabular}{|c|c|c|c|c|c|}
\hline No. & INDICADORES ESTRATEGICOS & UM & \multicolumn{3}{|c|}{ Cierre $31 / 3 / 10$} \\
\hline \multicolumn{6}{|c|}{ PERSPECTIVA FINANCIERA } \\
\hline \multirow{2}{*}{1} & \multirow{2}{*}{ Razón de rentabilidad económica } & & Plan & \multirow{2}{*}{ - } & 0.80 \\
\hline & & & Real & & 0.83 \\
\hline \multirow{2}{*}{2} & \multirow{2}{*}{ Razón de rentabilidad financiera } & & Plan & \multirow[b]{2}{*}{ - } & 0.63 \\
\hline & & & Real & & 0.57 \\
\hline \multirow[b]{2}{*}{3} & \multirow{2}{*}{ Tasa de ganancia } & \multirow[b]{2}{*}{$\%$} & Plan & \multirow[b]{2}{*}{ • } & 18.06 \\
\hline & & & Real & & 17.73 \\
\hline \multirow{2}{*}{4} & \multirow{2}{*}{ Índice de crecimiento de los ingresos totales } & \multirow[b]{2}{*}{$\%$} & Plan & \multirow[b]{2}{*}{ - } & 4.37 \\
\hline & & & Real & & 8.05 \\
\hline \multirow{2}{*}{5} & \multirow{2}{*}{ Índice de gastos totales por peso de ingreso } & \multirow{2}{*}{$\%$} & Plan & \multirow{2}{*}{ - } & 81.94 \\
\hline & & & Real & & 82.27 \\
\hline \multicolumn{6}{|c|}{ PERSPECTIVA CLIENTES } \\
\hline \multirow{2}{*}{6} & \multirow{2}{*}{ Índice de satisfacción del cliente } & & Plan & & 4.05 \\
\hline & & & Real & - & 3.92 \\
\hline & & & Plan & & 1.00 \\
\hline$r$ & Indice de desempeno de los procesos & & Real & - & 1.00 \\
\hline & PERPECTIVA PROCESOS INTERN & & & & \\
\hline & & & Plan & & 80.00 \\
\hline 8 & Nivel de satisfacción de los pedidos & $\%$ & Real & $\bullet$ & 37.73 \\
\hline 9 & Rotación de los inventarins de mercancías nara la venta & $\%$ & Plan & : & 55.08 \\
\hline y & 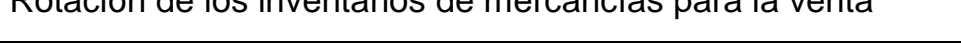 & $\%$ & Real & & 51.06 \\
\hline 10 & Índice de pérdidas por faltantes v mermas & $\%$ & Plan & 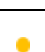 & 0.25 \\
\hline & & & Real & & 0.28 \\
\hline 11 & & & Plan & : & 100.00 \\
\hline 11 & Hechos extraordınarıos con participacion de empleados & $\%$ & Real & • & 100.00 \\
\hline 12 & Índice de ventas de mercancías y metro cuadrado & CuC/m² & Plan & & 13.00 \\
\hline 12 & 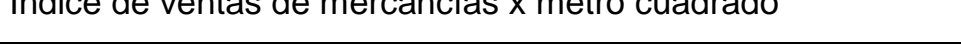 & $000 / m^{2}$ & Real & $\bullet$ & 8.49 \\
\hline 13 & & CuC/ha & Plan & & 20.00 \\
\hline & Indice del servicio de ventas de gastronomia $x$ banqueta & $\mathrm{CUC} / \mathrm{bq}$ & Real & $\bullet$ & 19.19 \\
\hline & PERSPECTIVA DE APRENDIZAJE Y CRE & OIMIENTO & & & \\
\hline & Relación calarin medin nroductividad & & Plan & & 1.03 \\
\hline 14 & Relacion salario medio productividad & & Real & - & 1.01 \\
\hline 15 & Índine do notinfonoión de olionto intorno & 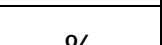 & Plan & & 95.0 \\
\hline 15 & Inaice de salistaccion del cliente interno & $\%$ & Real & - & 95.2 \\
\hline 16 & & $\%$ & Plan & & 2.00 \\
\hline 16 & I asa de fluctuacion laboral & $\%$ & Real & - & 0.64 \\
\hline 17 & Fvaluación de las acciones de canacitación & $\%$ & Plan & & 90.00 \\
\hline & & $\%$ & Real & - & 100.00 \\
\hline 18 & Fvaluación del desemneño & $\%$ & Plan & & 90.00 \\
\hline 18 & Evaluacion del desempeno & $\%$ & Real & - & 98.91 \\
\hline 19 & Índice del estado de la infraestructura constructiva y de & & Plan & & 0.91 \\
\hline 19 & equipos & & Real & - & 0.83 \\
\hline 20 & Cumplimiento del plan de mantenimiento de equipos & of & Plan & & 90.00 \\
\hline 20 & tecnológicos & $\%$ & Real & - & 75.23 \\
\hline 21 & Cumplimiento del presupuesto total de inversiones & $\%$ & Plan & & 100.00 \\
\hline & & & Real & - & 95.23 \\
\hline
\end{tabular}


Para la corrección de las desviaciones en los indicadores estratégicos se definió un plan de acción con un grupo de acciones correctivas que contribuyeron a mejorarlos.

Por la dinámica del CMI se determinó dejar de evaluar tres indicadores evaluados de excelente y con un comportamiento estable entre el segundo y tercer trimestre del 2011: índice de desempeño de los procesos, hechos extraordinarios con la participación de empleados y la tasa de fluctuación laboral.

El segundo trimestre del 2011, se incorporó al cuadro de mando el indicador de participación de productos nacionales en las ventas. El decrecimiento del indicador en el período, aunque sus cifras no son significativas y se mantuvo evaluado de bien, fue causado principalmente por una disminución en un $23 \%$ de los surtidos de proveedores locales.

Un indicador que durante los dos años y medio de evaluación presentó dificultades es el nivel de satisfacción de los pedidos (Figura 8). Este indicador con el plan de acción definido y otras acciones de la gerencia comercial fue posible su incremento en un $21.65 \%$ en el período y pasó de una evaluación de mal a regular. Una de las acciones que más contribuyó a los resultados fue la realización de gestiones directas por los comerciales en las distribuidoras.

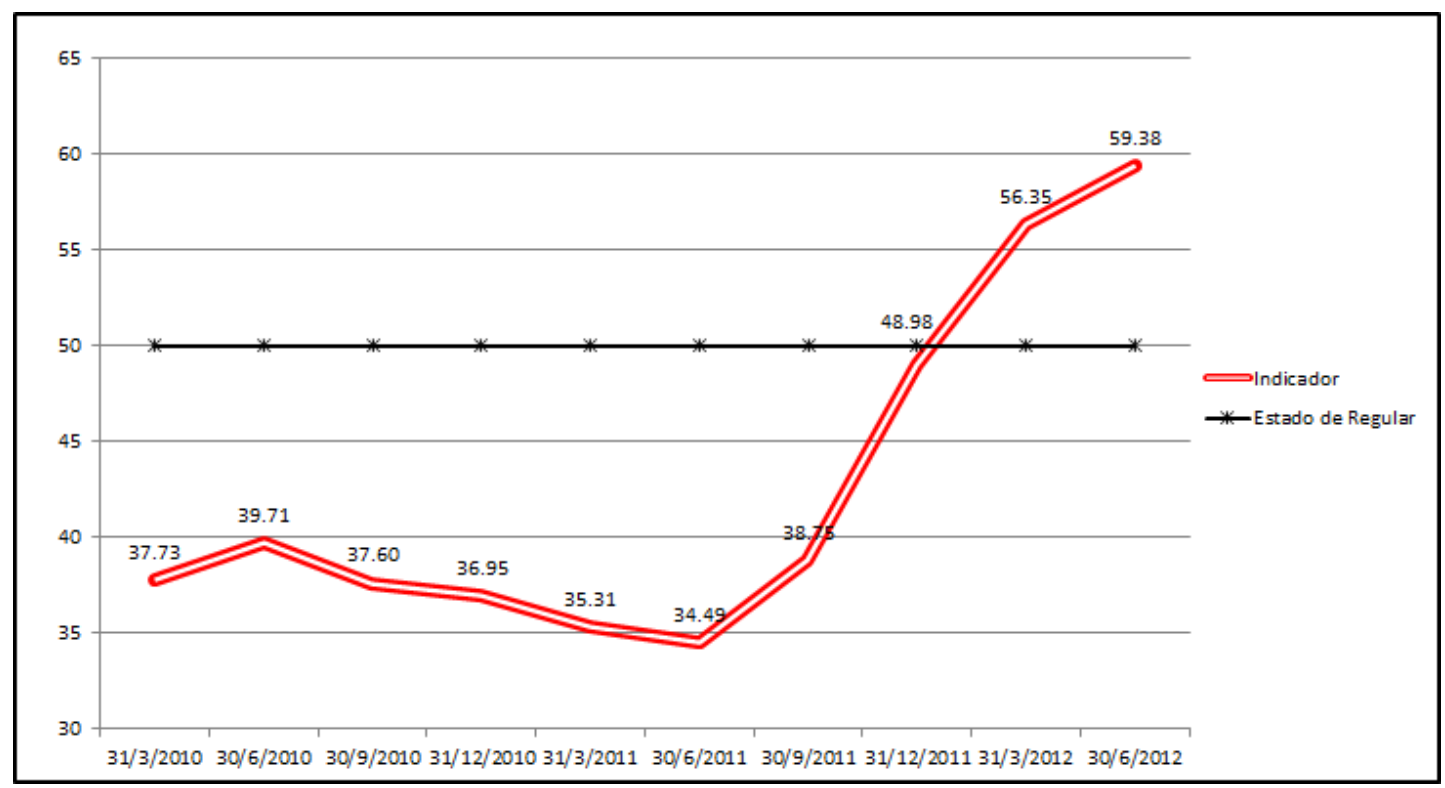

Figura 8 Evaluación del indicador nivel de satisfacción de los pedidos.

El indicador de nivel de satisfacción del cliente interno en los últimos seis meses del estudio mostró un decrecimiento de más de 6\% (Figura 9). Esta situación fue provocada por:

- Los locales de trabajo en general carecen de condiciones para prestar servicios sin que funcionen los aparatos de clima en los horarios pico.

- Falta de los insumos necesarios para el aseo de los trabajadores y las unidades

- Ausencia de toldo u otra alternativa permite que se produzcan resplandores molestos en los puestos de trabajo y en algunos casos deteriora la mercancía 
- Rotura de luminarias en los locales de trabajo sin posibilidades de reponer

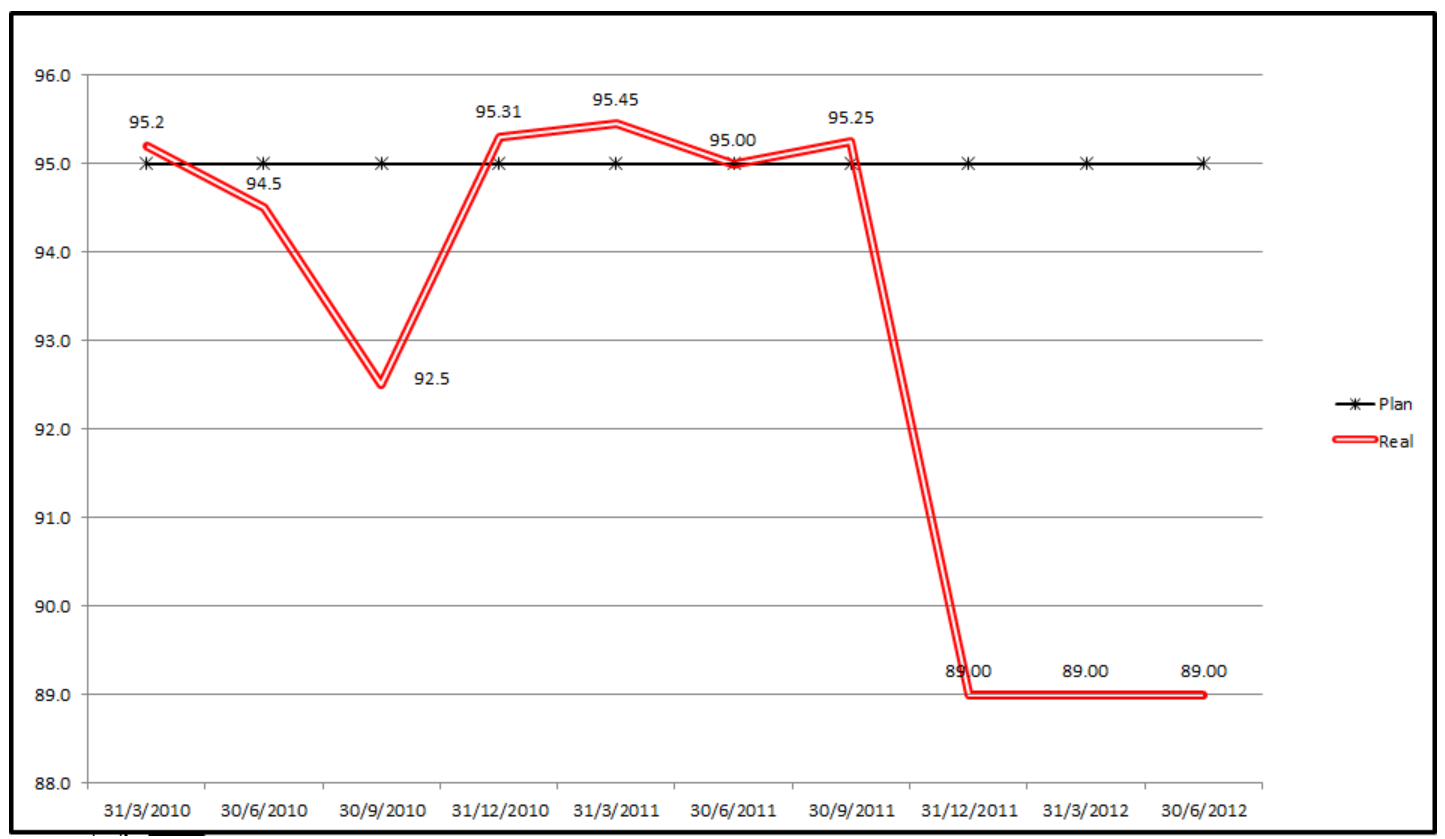

Figura 9. Evaluación del indicador índice de satisfacción del cliente interno.

Esta situación, determinó la necesidad de un plan de acciones correctivas para mejorar la satisfacción de los trabajadores en sus puestos de trabajo.

De forma general los indicadores estratégicos mejoraron su evaluación y la organización mejoró su eficiencia y eficacia a través de la implementación de la estrategia trazada. De un total de 25 indicadores iniciales, 12 pasaron a una evaluación de excelente de cinco iniciales (tres de estos indicadores se dejaron de evaluar en el intervalo de tiempo); en total los evaluados de bien y excelente finalmente son 20 y disminuyeron en dos los evaluados de mal por lo que sus resultados son favorables (Figura 10). El análisis del comportamiento de los indicadores de eficiencia y eficacia para evaluar el desempeño de los FCE que integraron el CMI permiten concluir que la implementación del procedimiento impacta positivamente en la gestión empresarial y resultó una herramienta eficaz para mantener la atención sobre la actuación de aquellos, que afectaba el cumplimiento de los objetivos estratégicos de la empresa.

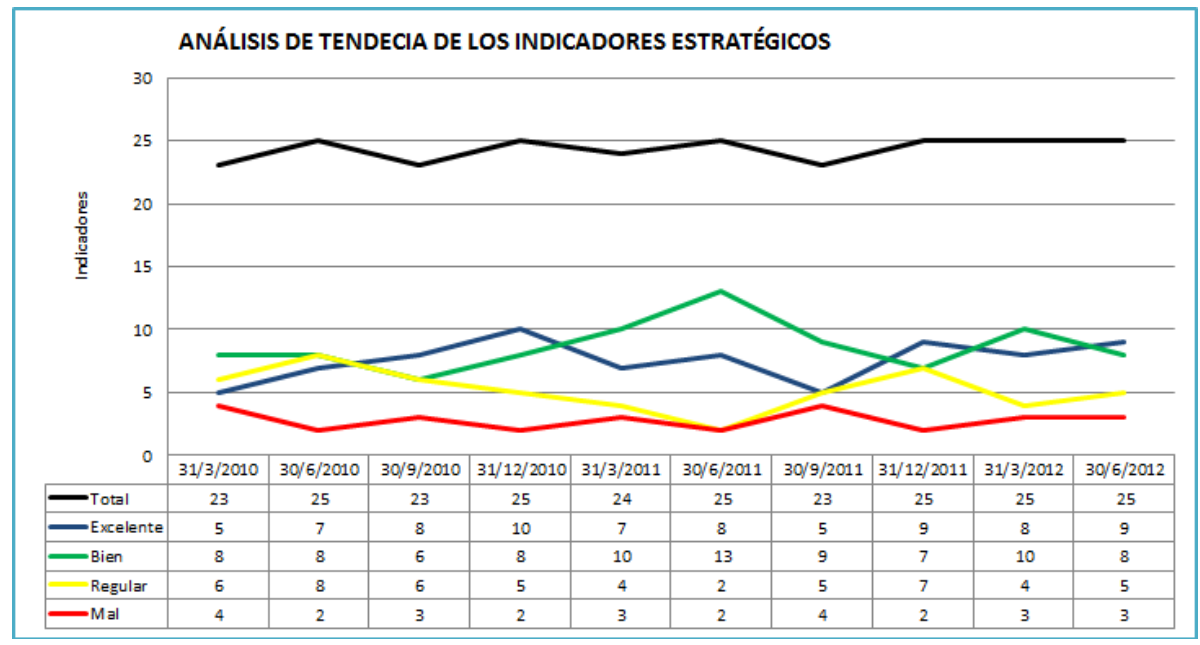

Figura 10. Análisis de los indicadores evaluados. 
Adicionalmente al presente caso de estudio, existen más de 15 aplicaciones que validan la pertinencia de los procedimientos establecidos desglosada en: tres empresas comercializadoras, un grupo empresarial de la construcción compuesto por ocho empresas y tres empresas constructoras, dos empresas de la industria alimentaria, dos empresas de servicio, un grupo hotelero y una universidad. La aplicación del procedimiento en empresas de tanta diversidad está dado también por su carácter flexible que le permite adecuarse a diversos entornos.

\section{Discusión}

En el marco del control de gestión se han realizado un conjunto de investigaciones, desde la década del 90 del siglo pasado, en diferentes sectores de la economía, que brindan un grupo de aportes importantes en la ciencia (Espino Valdés, Sánchez Sánchez, \& Aguilera Martínez, 2013; Martínez \& López, 2011; Nogueira Rivera, 2002; Porporato \& García, 2011; Sánchez Silva, 2007).

En este sentido, dentro de las herramientas del control de gestión, el CMI ha sido empleado con fuerza en los últimos 25 años en diferentes sectores y ramas de la economía. De hecho, en los últimos años se presentan muchas aplicaciones en el sector de los servicios, tales como: la salud pública (Naranjo Gil, 2010; Villalbí, 2007); los servicios universitarios (Moreno Freites, Eduardo Caballero, \& Bastidas, 2010); en empresas de software (González González \& Bermúdez Rodríguez, 2011). Las principales aportaciones del CMI, como herramienta de gestión son: centrarse en el control de los objetivos estratégicos de la organización, establecer una relación causa - efecto entre los indicadores de control seleccionados y el realizar el control a un número reducido de indicadores en tiempo real.

Desde su propia concepción el CMI se encuentra asociado a los procesos de planificación estratégica, sin embargo resultan escasos los intentos por asociar la gestión por procesos durante el proceso de planificación y generalmente es usado como herramienta de mejora (Medina León, Nogueira Rivera, Hernández Nariño, \& Viteri, 2010).

En la presente investigación se demuestra lo importante y factible de la incorporación de la filosofía de procesos a la planificación estratégica. La determinación de los procesos, su clasificación y formalización, de conjunto con la planificación estratégica; así como, la evaluación del alineamiento estratégico entre ambos y el uso del cuadro de mando integral permiten simplificar el camino y llevar al logro de la eficacia en las organizaciones.

\section{Conclusiones y Recomendaciones}

Con el propósito de analizar la situación del control de gestión en las organizaciones, y a partir de los resultados alcanzados, se puede concluir que existe la necesidad de trabajar en función de integrar las herramientas de control de gestión en las organizaciones. 
En la propuesta metodológica definida, como elemento novedoso, se integra la definición de los procesos desde la propia planificación estratégica, así como el diseño de la matriz de impacto de los procesos/objetivos estratégicos en función de la evaluación del alineamiento de los mismos. Se incluye además un procedimiento que facilita la definición de los mapas estratégicos en las organizaciones.

En la empresa comercializadora, establecida como caso de estudio durante el período investigado, a partir del despliegue de la estrategia y la realización de acciones correctivas durante su ejecución, se logra disminuir en uno los indicadores evaluados de mal e incrementar los evaluados de excelente en cuarto, logrando mejoras en su gestión.

La implementación del procedimiento propuesto, con su instrumental metodológico se logró en un grupo de organizaciones y los resultados alcanzados reflejan un perfeccionamiento en la gestión organizacional al mejorar sus indicadores de eficiencia y eficacia.

\section{Bibliografía}

Agudelo Tobón, L. F., \& Escobar Bolívar, J. (2010). Gestión por procesos. Medellín: ICONTEC.

Amo Baraybar, F. (2010). El cuadro de mando integral (The balanced scorecard). Barcelona: ESIC Editorial.

Boada Grau, J., \& Gil Ripoll, C. (2009). Gestión estratégica de recursos humanos como antecedente del balanced scorecard. Revista de Psicología del Trabajo y de las Organizaciones, 25(2).

Borchardt, M., Alfonso Sellito, M., \& Medeiros Pereira, G. (2007). Instrumento de avaliação para melhorias em processos organizacionais: caso do transporte coletivo rodoviário urbano de Porto Alegre. Produção, 17(2), 302-316.

Comas Rodríguez, R. (2013). Integración de herramientas de control de gestión para el alineamiento estratégico en el sistema empresarial cubano. Aplicación en empresas de Sancti Spiritus. (Tesis presentada en opción al grado científico de Doctor en Ciencias Técnicas), Universidad de Matanzas Camilo Cienfuegos, Matanzas

Del Canto, E. (2011). Gerencia estratégica y capital humano. Su prospectiva en los gobiernos locales en el contexto venezolano. Revista Ciencias Estratégicas., 19(26), 171-184.

Espino Valdés, A., Sánchez Sánchez, R., \& Aguilera Martínez, A. (2013). Procedimiento para el control de gestión en la Empresa de Campismo Popular de Villa Clara. Ingeniería Industrial, XXXIV(2), 227-236. 
González González, P., \& Bermúdez Rodríguez, T. (2011). Gerenciando intangibles en empresas de software aplicando el proceso de análisis por jerarquías y el cuadro de mando integral. Rev fac cienc econ, XIX(2), 89-104.

Kaplan, \& Norton, D. P. (2008). The execution premium. Integrando la estrategia y las operaciones para lograr ventajas competitivas (1ra ed.). Barcelona: Ediciones Deusto.

Martínez, E. A., \& López, M. I. (2011). Control de gestión en unidades de producción de ganadería de doble propósito. Visión Gerencial, 10(2), 325-340.

Medina León, A., Nogueira Rivera, D., Hernández Nariño, A., \& Viteri, J. (2010). Relevancia de la gestión por procesos en la planificación estratégica y la mejora continua. Revista Eídos, 2.

Moreno Freites, Z., Eduardo Caballero, A., \& Bastidas, E. (2010). Planificación estratégica y el cuadro de mando integral: herramientas de gestión para mejora la prestación de los servicios universitarios. TEACS, 3(5), 9-23.

Naranjo Gil, D. (2010). El uso del cuadro de mando integral y del presupuesto en la gestión estratégica de los hospitales públicos. Gaceta Sanitaria, 24(3), 220-224.

Nogueira Rivera, D. (2002). Modelo conceptual y herramientas de apoyo para potenciar el control de gestión en las empresas cubanas. (Tesis presentada en opción al grado científico de Doctor en Ciencias Técnicas), Universidad de Matanzas "Camilo Cienfuegos", Matanzas. (Tesis en el repositorio de Ingeniería Industrial)

Paladino, B., \& Williams, N. (2008). Moving Strategy Forward: Merging the Balanced Scorecard and Business Intelligence. Business Performance Management Magazine, 6(2), 12.

Porporato, M., \& García, N. (2011). Sistemas de control de gestión: un estudio exploratorio de su efecto sobre el desempeño organizacional. Academia. Revista Latinoamericana de Administración, 47, 61-77.

Sánchez Silva, E. (2007). Diseño de un sistema de Control de Gestión para el departamento de movilización de la ilustre Municipalidad de Maipú. http://www.cybertesis.cl/tesis/uchile/2007/sanchez_e/html/index-frames.html

Shpilberg, D., Berez, S., Puryear, R., \& Shah, S. (2007). Avoiding the alignment trap in IT. MIT Sloan Management Review, 49(1), 51.

Villalbí, J. (2007). El Cuadro de Mando Integral como instrumento de dirección en una organización de salud pública. Gaceta Sanitaria, 21(1), 60-65. 